\title{
QTe prolongation with concurrent use of azithromycin and diltiazem in an old female patient: a case report
}

\author{
Lama Soubra $^{1}$, Ali Mroueh ${ }^{2}$, Samer Kabbani $^{3}$
}

\begin{abstract}
${ }^{1}$ Department of pharmacy practice, Department of Pharmacy, Beirut Arab University, Beirut, Lebanon, ${ }^{2}$ Department of Cardiology, Faculty of Medical Sciences, The Lebanese University, Beirut, Lebanon, ${ }^{3}$ Department of Cardiology, Rafic Hariri University Hospital, Beirut, Lebanon
\end{abstract}

Received: 27 November 2013 Accepted: 15 December 2013

\section{*Correspondence to:}

Dr. Lama Soubra,

Email: 1.soubra02@bau.edu.lb, soubralama@hotmail.com

(C) 2014 Soubra L et al. This is an openaccess article distributed under the terms of the Creative Commons Attribution NonCommercial License, which permits unrestricted non-commercial use, distribution, and reproduction in any medium, provided the original work is properly cited.

\begin{abstract}
Azithromycin is widely prescribed for the treatment of respiratory tract infections. Incidence of corrected QT interval (QTc) prolongation and cardiac arrest has not been reported after concomitant administration of azithromycin and diltiazem. Here we present a 69-year-old female patient who developed profound QTc prolongation and cardiac arrest after three days of concomitant administration of azithromycin and diltiazem. The patient was successfully resuscitated, intravenous magnesium was given and azithromycin therapy was discontinued. The QTc interval dropped to $412 \mathrm{~ms} 24$ hours after azithromycin discontinuation. One week later, the patient was discharged home after full recovery. This case illustrates a possible drug interaction between azithromycin and Pglycoprotein inhibitor drugs and/or drugs having an effect on cardiac repolarization.
\end{abstract}

Keywords: Azithromycin, Diltiazem, Drug Interaction, QTc prolongation, Torsade de pointes, Cardiac arrest

\section{INTRODUCTION}

Macrolide antibiotics are used to treat upper respiratory tract infections, community acquired pneumonia and other infections. Prolonged cardiac muscle repolarization leading to ventricular arrhythmias, notably torsade de pointes and sudden death are well described as adverse reactions and are common to all macrolides.,

Until recently, azithromycin has been considered the safest of the macrolides in terms of overall toxicity, as it neither undergoes CYP3A4 metabolism nor inhibits CYP3A4 to any clinically meaningful degree, and therefore does not interfere with commonly prescribed medications that undergo CYP3A4 metabolism. Azithromycin was also thought to have lower pro- arrhythmic potential than other macrolides, in vitro. ${ }^{3,4}$ Despite these properties, corrected QT interval (QTc) prolongation and torsade de pointes were reported with azithromycin use. ${ }^{4-6}$ A recent study concluded that azithromycin therapy increased the risk of cardiovascular death. $^{7}$ Azithromycin cardio-toxicity was believed to be more pronounced among patients with a high baseline risk for cardiovascular disease ${ }^{1,7,8}$ and at higher serum levels. ${ }^{9}$

To the best of our knowledge, there are no reported cases of QTc prolongation and torsade de pointes induced by the concurrent use of azithromycin and diltiazem. We describe the first documented case of QTc prolongation and torsade de pointes possibly linked to an interaction between these two drugs. 


\section{CASE REPORT}

A 69-year-old female patient presented to the emergency department (ED) for a survived cardiac arrest that occurred at home. Thirty minutes after her arrival to the $\mathrm{ED}$, she developed a second episode of cardiac arrest. The patient received supportive care and was transferred to the cardiac care unit for post-cardiac arrest management after having restored her hemodynamic stability. The patient had no personal or family history of long QT syndrome and no reported history of allergies. Her medical history was remarkable for chronic atrial fibrillation and hypertension treated with diltiazem XR $300 \mathrm{mg}$ PO once daily and Warfarin $2 \mathrm{mg}$ PO once daily since 5 years, diabetes mellitus treated with gliclazide MR $60 \mathrm{mg}$ PO once daily since 10 years and hypothyroidism treated with levothyroxine $100 \mathrm{mcg}$ PO once daily since 8 years. Three days prior to ED admission she had a lower respiratory tract infection which was treated with azithromycin $500 \mathrm{mg}$ PO once at the first day, followed by $250 \mathrm{mg}$ PO once daily. Initial laboratory testing results were as follows: Sodium, 143 Meq/l; Potassium, 4.05 Meq/L; Chloride, $104 \mathrm{Meq} / \mathrm{L}$; Bicarbonates, $36 \mathrm{Meq} / \mathrm{L} ; \quad$ Calcium $8.7 \mathrm{Meq} / \mathrm{L}$; Magnesium, $1.5 \mathrm{Meq} / \mathrm{L}$; glucose $131 \mathrm{mg} / \mathrm{dL}$; White Blood Cell count, $7.3 \times 10^{3}, 65 \%$ of which were neutrophils; and Hemoglobin level, $11.6 \mathrm{~g} / \mathrm{dl}$. Arterial blood gases $(\mathrm{ABGs})$ showed $\mathrm{pH}=7.49, \mathrm{PaCO} 2=50$ $\mathrm{mmHg}, \mathrm{PaO} 2=82 \mathrm{mmHg}, \mathrm{HCO} 3-=32 \mathrm{Meq} / \mathrm{L}$ and $\mathrm{SaO} 2$ $=96 \%$. Her cardiac enzymes, $\mathrm{HBA}_{1} \mathrm{C}$ and Thyroid Stimulating Hormone (TSH) were within the normal limits. The electrocardiogram (ECG) showed a significantly prolonged QTc interval of $666 \mathrm{~ms}$ with torsade de pointes rhythm and no ST-T changes (Figure 1 and Figure 2). A brain computed tomography scan showed an old stroke with no active bleeding. The azithromycin therapy was discontinued and $2 \mathrm{~g}$ of intravenous magnesium was given to the patient. The QTc interval dropped to $412 \mathrm{~ms} 24$ hours after azithromycin discontinuation (Figure 2). After that baseline atrial fibrillation with back-to-normal QT interval was achieved (Figure 3). One week later, the patient was discharged home after full recovery.

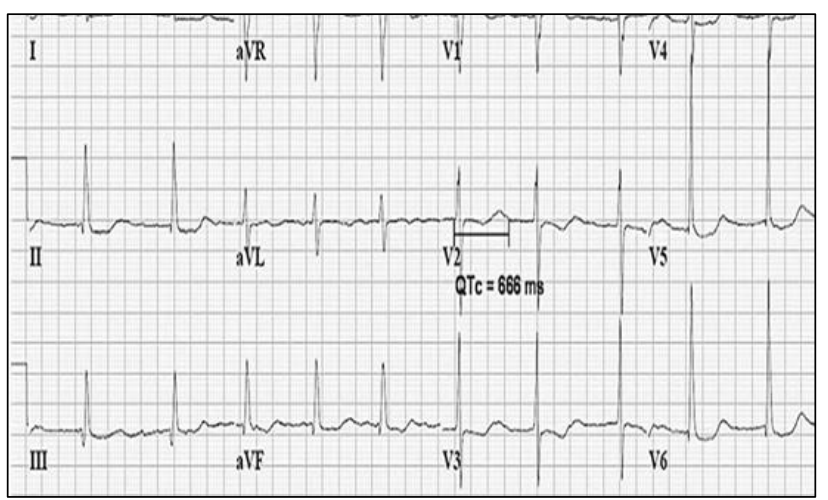

Figure 1: ECG showing markedly prolonged QT interval.

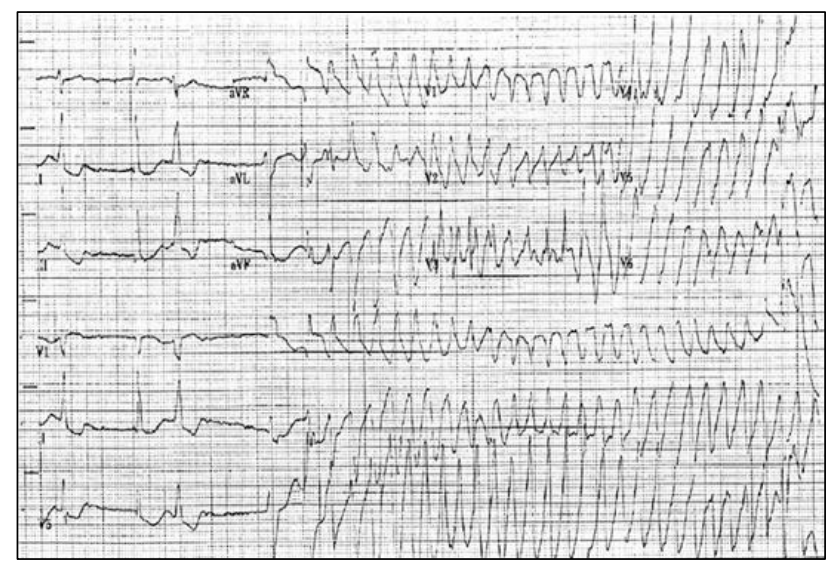

Figure 2: ECG showing torsade de pointes.

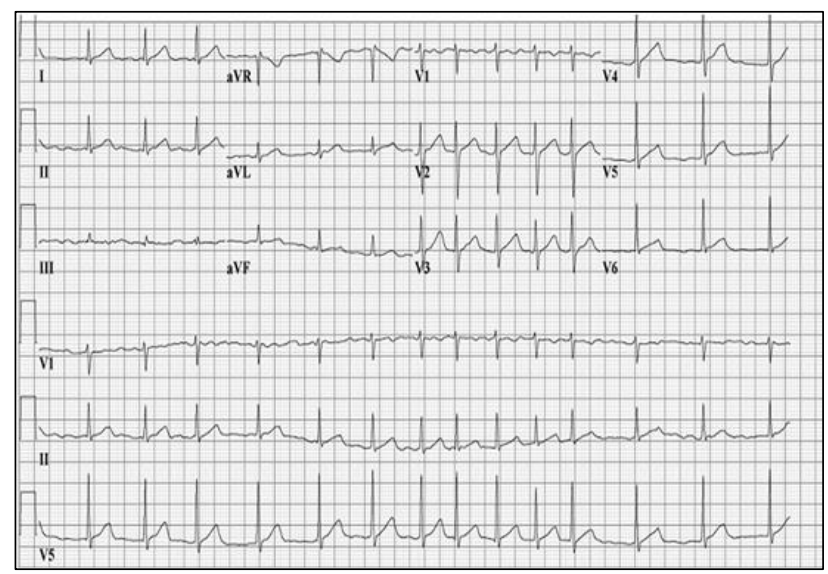

Figure 3: ECG showing atrial fibrillation with backto-normal QT interval.

\section{DISCUSSION}

The patient discussed here had a markedly prolonged QTc interval after three days of concurrent use of azithromycin and diltiazem. We believe that our patient's case illustrates an interaction between oral azithromycin and oral diltiazem, as evidenced by the temporal relationship between QTc prolongation with the administration of oral azithromycin and the return toward the normal after azithromycin discontinuation.

In search of a cause for QTc prolongation, inherited (primary) and acquired disorders (secondary) must be considered. Recent research has elucidated genetics and molecular pathogenesis of congenital (primary) long QTc syndrome and at least six forms of the disorder have been attributed to mutations in cardiac ion channels. ${ }^{10,11}$ The secondary (acquired) form of QTc syndromes is attributed to metabolic abnormalities (e.g. hypokalemia), medical conditions (e.g., myocarditis, subarachnoid hemorrhage), and drugs that prolong ventricular repolarization. ${ }^{11,12}$ Irrespective of the cause, markedly prolonged QTc intervals, such in this case, may confer an increased risk 
of sudden death due to polymorphic ventricular tachycardia, particularly of the torsade de pointes variant. $^{13,14}$ In the patient reported here, an acquired (secondary) cause of QTc prolongation was assumed since the patient had no history of inherited long QTc interval. This patient had no electrolytes disturbances, no metabolic abnormalities, no significant structural heart disease, and no clinical or laboratory evidence of ongoing cardiac ischemia or stroke. However, the patient reported here, received two drugs with a potential to affect cardiac repolarization: diltiazem and azithromycin.

The concurrent use of multiple drugs with a risk to prolong QTc interval, even if this risk is limited or minimal, was well reported as a predisposing factor for drug-induced QTc interval prolongation and torsade de pointes secondary to a pharmacodynamics based (PDbased) interaction. ${ }^{15}$ Diltiazem affects cardiac repolarization by blocking the rapid component of the delayed rectifier $\mathrm{K}+$ current $\left(\mathrm{I}_{\mathrm{Kr}}\right)$ channel. Diltiazem is less likely to cause Early After depolarization (EAD), or to increase dispersion of ventricular repolarization and therefore to cause torsade de pointes. ${ }^{16}$ Azithromycin can also disturb cardiac repolarization and prolong the QT interval. ${ }^{3,13}$ The exact mechanism of QTc prolongation and the resulting torsade de pointes associated with azithromycin is not well understood. Since azithromycin and erythromycin are both azalide antibiotics, they probably have similar properties and, therefore similar mechanisms of action. In vitro and in vivo studies revealed that erythromycin prolongs cardiac repolarization primarily by blockade of the $\mathrm{I}_{\mathrm{Kr}}$ channel. It also causes EAD and triggered activity in $M$ cells, resulting in increased dispersions of refractoriness in ventricular tissue and torsade de pointes. ${ }^{15,17}$ Since azithromycin and diltiazem both act on the $\mathrm{I}_{\mathrm{Kr}}$, therefore they may have had additive effects on the potential action duration which resulted in QTc interval prolongation and Torsade de pointes in this patient.

Higher serum concentrations of a QT-prolonging drug have also been well characterized as a risk for QTc prolongation and its variant torsade de pointes. ${ }^{15-18}$ Many factors may contribute to higher drug serum concentrations such as higher than average drug dosage and decreased clearance. Decreased drug clearance is observed in patients with liver or kidney impairment and in patients taking agents that may interfere with their clearance.

This patient received azithromycin and diltiazem in regular doses. Moreover, this patient does not have liver or kidney impairment. However, the concurrent use of azithromycin and diltiazem may have led to an increased serum concentration of azithromycin and /or diltiazem. To explore the possible mechanism for this interaction, the effects of these agents on their respective clearance should be considered. Diltiazem is mainly metabolized in the liver through the CYP $3 \mathrm{~A} 4$. It is an inhibitor of the CYP 3A4 and the P-glycoprotein (P-gp) efflux pump. ${ }^{19,20}$ Azithromycin has minimal hepatic metabolism and does not interact with the CYP system. ${ }^{13,21,22}$ It is metabolized to an inactive metabolite via $\mathrm{N}$-demethylation through other routes independent of the CYP system. Azithromycin is mainly excreted unchanged in the bile with subsequent elimination in the stool. It has been suggested that the trans-intestinal excretion involving Pgp efflux pump may be the primary route of elimination of unchanged azithromycin. ${ }^{23-25}$ Since azithromycin does not interact with the CYP 3A4 system, an interaction between azithromycin and diltiazem at this level is unlikely. However, by inhibiting the P-gp efflux pump, diltiazem could have interfered with azithromycin clearance thereby increasing azithromycin serum concentrations and its effects. Therefore, QTc prolongation and Torsade de pointes observed in this patient may have been caused by a pharmacokineticbased (PK-based) interaction between azithromycin and diltiazem.

The patients' other medications were not reported to prolong QTc nor to interfere with azithromycin or diltiazem clearance. The patient thus had no other inciting drug interactions that could have caused the QTc interval prolongation and torsade de pointes.

Limitations of our case are: first, azithromycin and macrolides have shown to be independent risk factors for torsade de pointes; ${ }^{15}$ second, the lack of any report of an obvious mechanism for this diltiazem-azithromycin interaction; third the lack of azithromycin blood level measurement at the time of the event; and finally the presence of other predisposing factors (old age and female gender) in this patient that confer increased vulnerability for macrolide induced QT prolongation and torsade de pointes. ${ }^{15,18}$ In addition, macrolides occasionally unmask an inherited long QT syndrome; ${ }^{26}$ therefore, genetic vulnerability may also play a part.

QTc prolongation and Torsade de pointes could have been prevented in this patient. This could have been done by a clinical pharmacist and/or clinician through identifying predisposing factors in this patient; avoiding concurrent use of diltiazem and azithromycin and recognizing potential drug-drug interactions with these agents. Although consideration of all these areas will likely minimize the patient's rate of drug-induced QTc prolongation, nothing can eliminate a patient's risk completely. It is therefore recommended to monitor closely patients receiving drugs noted to cause QTc prolongation, especially those who are predisposed. This could be done by obtaining and measuring QTc interval regularly.

\section{CONCLUSION}

Diltiazem may increase Azithromycin toxicity by inhibiting P-glycoprotein responsible for its clearance and/or by a direct pharmacodynamic interaction. This would cause an increased myocardial repolarization time manifested by a prolonged QTc interval on ECG resulting in Torsade de pointes. The co-administration of 
azithromycin and a P-glycoprotein inhibitors or drugs that affect cardiac repolarization should, therefore, be approached with caution, especially in patients who has other predisposing factors for QTc prolongation and torsade de pointes such as age $>65$ year-old, and female gender. Close monitoring for evidence of QTc prolongation and Torsade de pointes is also advisable. Further investigation into the mechanism by which these two drugs interacted may be indicated.

Funding: No funding sources

Conflict of interest: None declared

Ethical approval: Not required

\section{REFERENCES}

1. Russo V, Puzio G, and Siniscalchi N. Azithromycininduced QT prolongation in elderly patient. Acta Biomed. 2006;77(1):30-2.

2. Ohtani H, Taninaka C, Hanada E, Kotaki H, Sato H, Sawada $\mathrm{Y}$ et al. Comparative pharmacodynamic analysis of Q-T interval prolongation induced by the macrolides clarithromycin, roxithromycin, and azithromycin in rats. Antimicrob Agents Chemother. 2000;44(10):2630-7.

3. Milberg P, Eckardt L, Bruns HJ, Biertz J, Ramtin S, Reinsch $\mathrm{N}$ et al. Divergent proarrhythmic potential of macrolide antibiotics despite similar QT prolongation: fast phase 3 repolarization prevents early afterdepolarizations and torsade de pointes. J Pharmacol Exp Ther. 2002;303(1):218-25.

4. Federal Drug Comission. FDA/PhRMA task force to assess QT risk by preclinical markers. Pink sheet. 1999:61:15-16.

5. Kim MH, Berkowitz $\mathrm{C}$, and Trohman RG. Polymorphic ventricular tachycardia with a normal QT interval following azithromycin. Pacing Clin Electrophysiol. 2005;28(11):1221-2.

6. Kezerashvili A, Khattak H, Barsky A, Nazari R and Fischer JD. Azithromycin as a cause of QT-interval prolongation and torsade de pointes in the absence of other known precipitating factors. J Interv Card Electrophysiol. 2007;18(3):243-6.

7. Wayne R, Murray K, Arbogast G and Stein M. Azithromycin and the Risk of Cardiovascular Death. New England Journal of Medicine. 2012;366(20):1881-90.

8. Matsunaga, N., Y. Oki, and A. Prigollini. A case of QT-interval prolongation precipitated by azithromycin. N Z Med J. 2003;116:1185.

9. Tilelli JA, Smith KM, and Pettignano R. Lifethreatening bradyarrhythmia after massive azithromycin overdose. Pharmacotherapy. 2006;26(1):147-50.

10. Ackerman, M.J. The Long QT Syndrome: Ion Channel Diseases of the Heart. Mayo Clinic proceedings. Mayo Clinic. 1998;73(3):250-69.

11. Viskin S. Long QT syndromes and torsade de pointes. Lancet. 1999;354:1625-33.
12. Laakso M, Aberg A, Savola J, Pentikäinen PJ and Pyörälä K. Diseases and drugs causing prolongation of the QT interval. Am J Cardiol. 1987;59(8):862-5.

13. Owens, R.C. Risk Assessment for Antimicrobial Agent-Induced QTc Interval Prolongation and Torsades de Pointes. Pharmacotherapy: The Journal of Human Pharmacology and Drug Therapy. 2001;21(3):301-319.

14. Haverkamp W, Eckardt L, Monnig G, Schulze-Bahr E, Wedekind $\mathrm{H}$, Kirchhof $\mathrm{P}$ et al. Clinical aspects of ventricular arrhythmias associated with QT prolongation. European Heart Journal Supplements. 2001;3(suppl K):K81-8.

15. Nachimuthu S, Assar MD, and Schussler JM. Druginduced QT interval prolongation: mechanisms and clinical management. Therapeutic Advances in Drug Safety. 2012;3(5):241-53.

16. Belardinelli L, Antzelevitch C, and Vos MA. Assessing predictors of drug-induced torsade de pointes. Trends in pharmacological sciences. 2003;24(12):619-25.

17. Katapadi K, Kostandy G, Katapadi M, Hussain KM and Schifter D. A review of erythromycin-induced malignant tachyarrhythmia--torsade de pointes. A case report. Angiology. 1997;48(9):821-6.

18. Gupta A, Lawrence A, Krishnan K, Kavinsky C, Trohman R. Current concepts in the mechanisms and management of drug- induced QTc prolongation and Torsade de pointes. Amer heart J. 2007;153(6):8919.

19. Thomas AR, Chan LN, Bauman JL and Olopade CO. Prolongation of the QT interval related to cisapridediltiazem interaction. Pharmacotherapy. 1998;18(2):381-5.

20. Horn JR, Hansten P. Drug interactions with digoxin: the role of P-glycoprotein. Pharmacy times. 2004;Oct:114-5.

21. Westphal JF. Macrolide - induced clinically relevant drug interactions with cytochrome P-450A (CYP) 3A4: an update focused on clarithromycin, azithromycin and dirithromycin. $\mathrm{Br} \quad \mathrm{J}$ Clin Pharmacol. 2000;50(4):285-95.

22. Galetin A, Burt H, Gibbons L and Houston JB. Prediction of time-dependent CYP3A4 drug-drug interactions: impact of enzyme degradation, parallel elimination pathways, and intestinal inhibition. Drug Metab Dispos. 2006;34(1):166-75.

23. Robert L P, Ruscin JM, Fish D and LaPointe M. Possible Interaction Between Intravenous Azithromycin and Oral Cyclosporine. Pharmacotherapy. 2001;21(11):1436-43.

24. Pachot JI, Botham RP, Haegele KD, Hwang K. Experimental estimation of the role of $\mathrm{P}$ Glycoprotein in the pharmacokinetic behaviour of telithromycin, a novel ketolide, in comparison with roxithromycin and other macrolides using the Caco-2 cell model. J Pharm Pharm Sci. 2003Jan-Apr;6(1):112.

25. Sugie M, Asakura E, Zhao LH, Torita S, Nadai M, Baba K and al. Possible Involvement of the Drug 
Transporters P Glycoprotein and Multidrug Resistance-Associated Protein Mrp2 in Disposition of AzithromycinAntimicrob Agents Chemother. 2004March;48(3):809-14.

26. De Ponti F, Poluzzi E, Cavalli A, Recanatini M, and Montanaro N. Safety of Non-Antiarrhythmic Drugs that Prolong the QT Interval or Induce Torsade de Pointes. Drug Safety, 2002;25(4):263-86.

doi:10.5455/2319-2003.ijbcp20140244

Cite this article as: Soubra L, Mroueh A, Kabbani S. QTc prolongation with concurrent use of azithromycin and diltiazem in an old female patient: a case report.

Int J Basic Clin Pharmacol 2014;3:242-6. 